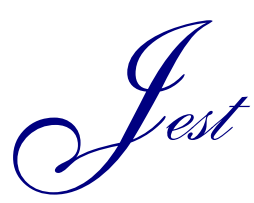

Research Article

\title{
Identification of Sine, Squire, Triangle and Sawtooth Waveforms with Uniform White and Inverse F Noises by Adaptive Neuro-Fuzzy Interface System
}

\author{
G. Georgiev ${ }^{1, *}$, I. Balabanova ${ }^{1}$, P. Kogias ${ }^{2}$, S. Sadinov ${ }^{3}$ and S. Kostadinova ${ }^{3}$ \\ ${ }^{1}$ Technical University of Gabrovo, Bulgaria, \\ ${ }^{2}$ Dpt of Electrical Engineering, Eastern Macedonia and Thrace Institute of Technology, Kavala, Greece, \\ ${ }^{3}$ Technical University of Varna, Bulgaria
}

Received 15 December 2017; Accepted 18 July 2018

\begin{abstract}
In this paper the structures of Adaptive Neuro-fuzzy interface system (ANFIS) are studied for noise identification. The system's structures are analyzed for different types of membership functions applied for input variables with root mean square errors variation. Hybrid algorithm and back propagation algorithm are applied. The input data are obtained through system simulation based on LabVIEW system design platform and development environment. The choice of ANFIS structure is based on the training results and minimum RMSE for identification of the signals with uniform white and inverse F Noises. Therefore, "gbellmf" membership function for input data variables is chosen. The accuracy classification is obtained at $100 \%$.
\end{abstract}

Keywords: LabVIEW simulation, signal identification, adaptive neuro-fuzzy interface system, membership function, classification accuracy.

\section{Introduction}

Adaptive neuron-fuzzy interface systems (ANFIS) emulate the human artificial intelligence by combining the advantages of artificial neural networks mathematical models and fuzzy logic.

ANFIS have a number of applications in solving the complex problems in the areas of computer science, industry, technology, medicine, in the mobile communication systems for detection of unauthorized user access, forecasting product demand for $3 \mathrm{G}, 4 \mathrm{G}$ and $5 \mathrm{G}$ mobile phones [1, 2].

Neural fuzzy systems find their main implementation in control systems in industrial process units and processes, using their advantages for modeling of nonlinear systems by linear interpolation models as accomplishment the PID controllers in the event of changes in the state of airplanes, control of origin and destination, industrial robot machines etc. [3-5].

The successful classification of ECG signals in the field of the medical diagnostics is in the basis of the detection of heart attacks and cardiovascular abnormalities and disorders [6]. According to the researches, the most common approach to solving the problem is in using of fuzzy neural interface models.

An example of classification of six types of electrocardiogram signals with normal sinus rhythm (NSR), premature ventricular contraction (PVC), atrial premature contraction (APC), Ventricular Tachycardia (VT), Ventricular Fibrillation (VF) and Supraventricular Tachycardia (SVT) is given

*E-mail address: givanow@abv.bg

ISSN: $1791-2377$ @ 2018 Eastern Macedonia and Thrace Institute of Technology. All rights reserved. doi:10.25103/jestr.113.17 in [7]. In other research it is presented a new QRS complex geometric feature extraction technique and its application for electrocardiogram (ECG) supervised hybrid (fusion) beattype classification [8]. An interesting method is proposed for automated diagnostics system for electrocardiography of arrhythmias, including the denoising module, the classifier module and the optimization module. In first of the modules, the stationary wavelet transform is applied for electrocardiograms signals noise reduction, the systems classification module is based on ANFIS and "the cuckoo optimization algorithm" is used in the optimization module [9]. There is provided a system for the removal of the non-linear artifacts from respiratory signal, measured by MEMS based accelerometer sensor, through innovative ANFIS-based adaptive filter. The system combines an adaptive filter, integrating the Least Mean Square (LMS) and Recursive Least Square (RLS) algorithms, and ANFIS architecture [10]. In a similar study, to reduce the noise present in the pure respiratory signal is carried out a comparison of the an adaptive filtering based on ANFIS and filtering trough LMS and RLS [11].

An intelligent approach for adaptive filtering is used in signal processing for removing the noise from audio speech signals [12]. Another application of ANFIS is in modern technologies associated with the improving the quality of human speech perception in hearing aids where there is a need for sound classification algorithms [13].

Brain-computer interfacing is an emerging field of research where signals extracted from the human brain are used for decision making and generation of control signals. Selection of the right classifier to detect the mental states from electroencephalography (EEG) signal is an open area of research because of the signal's non-stationary and Ergodic nature. Though neural network based classifiers, like ANFIS, act efficiently, to deal with the uncertainties involved in EEG signals [14]. Another study examines as a 
G. Georgiev, I. Balabanova, P. Kogias, S. Sadinov and S. Kostadinova/

Journal of Engineering Science and Technology Review 11 (3) (2018) 128-132

object the snore related sounds (SRSs) for detection of sleep apnea / hypopnea syndrome (SAHS), as indicator of specific sleep disorders [15]. Of interest is the application of ANFIS in signal processing systems for energy production from renewable energy sources such as wind turbines and others. The essence of the method is calculating of the noise level of the wind turbine as regards of the wind speed and sound frequency trough adaptive neural-fuzzy system [16].

Another area of application of neural-fuzzy interface systems is for prediction and evaluation of the noise of traffic flows of vehicles in various urban environments based on the traffic flow, vehicle speed and honking [17].

Filtering signals, identification, reduction and study of the effects of existing noise is a major problem in data transmission in telecommunication systems. This report is aimed at developing an innovative approach to identifying noise in analog and digital signals with the use of ANFIS architecture. The results obtained in the study allow for successful implementation of synthesized neural-fuzzy model as a system module for noise identification, securing data transmission and quality of service in modern telemetric communication systems.

\section{Identification of Signals with Superimposed Noises}

\subsection{LabVIEW simulation data}

Sine, Square, Triangle and Sawtooth waveforms with Uniform White Noise and Inverse F Noise are simulated based on LabVIEW system design platform and development environment. The system set parameters are:

- Signals wveforms - Sine, Square, Triangle and Sawtooth waveforms;

- Signals parameters - frequency (Hz): 10,1; Amplitude: 1; Phase: 0;

- Superimposed noises - Umiform White Noise and Inverse F Noise;

- Noises parameters - Noise amplitude: 0,6; Seed Number: -1 ;

- Simulation parameters - Samples per second (Hz): 1000; Simulate acquisition timing; Number of samples: 300 .

It is formed an input data set of 600 etalons containing values of four independent predictive variables:

1. Sine waveform with noise - labeled "Sine";

2. Square waveform with noise - labeled "Square";

3. Triangle waveform with noise - labeled "Triangle";

4. Sawtooth waveform with noise - labeled "Sawtooth". Two classification groups are defined:

- class №1: Waveforms with Uniform White Noise - labeled "UWN";

- class №2: Waveforms with Inverse F Noise - labeled "IFN".

The inputs are distributed in two to one ratio, approximately $67 \%$ for training and $33 \%$ for testing the neural fuzzy classifier (400 training and 200 test samples).

\subsection{Results in the training of an adaptive neural fuzzy interface system for identifying signals with superimposed noises}

There has been training ANFIS architecture (Fig. 1) by a hybrid algorithm combining the method of "Least squares" and "Back error propagation" method and algorithm with "Back propagation of error". Attached are the following input variables, respectively, "Sine", "Square", "Triangle" and "Sawtooth" waveforms with superimposed noise. This paper examines the variation of error in training, RMSE (Root Mean Square Error) in specified algorithms for different types of membership functions of input variables in sequential order "trimf", "trapmf", "gbellmf", "gaussmf", "gauss2mf", "pimf"," dsigmf" and "psigmf". The output classes are defined by membership functions of the output variable from categorical type of membership for the constant type of output variable, respectively, values "1" to the class "UWN" and "2" for the class "IFN". With respect to the class №1 for correctly classified are accepted ANFIS calculated variables in the range of 0.5 to 1.5 , while for class №2 in the range of 1.5 to 2.5 . A minimum value of RMSE = 0.062289 is found by observing the results of the training, obtained for bell type ("gbell") of membership functions of the input variables, for hybrid learning algorithm. Variation of the error is represented in Fig. 2.

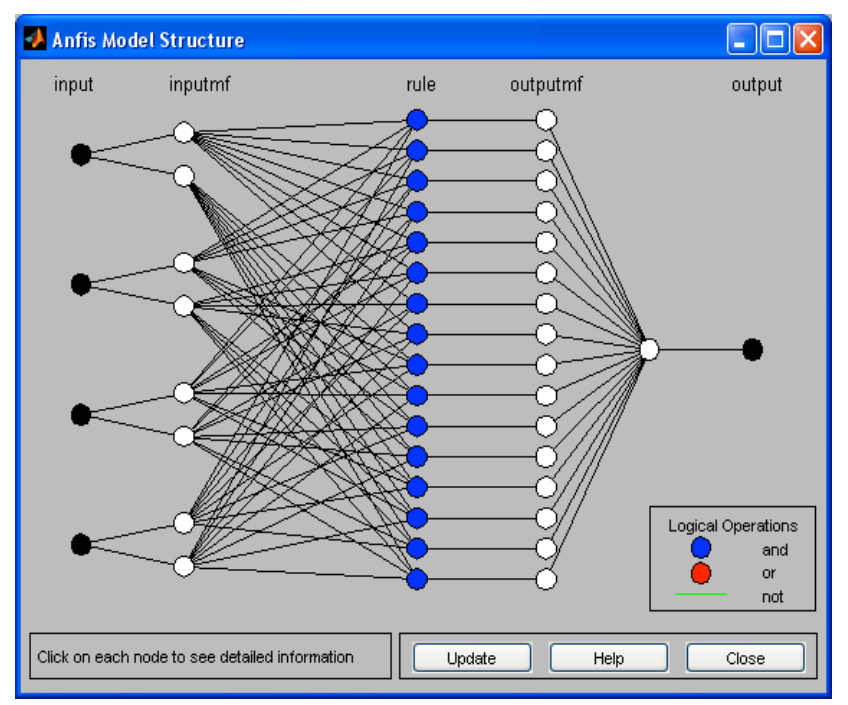

Fig. 1. ANFIS classifier

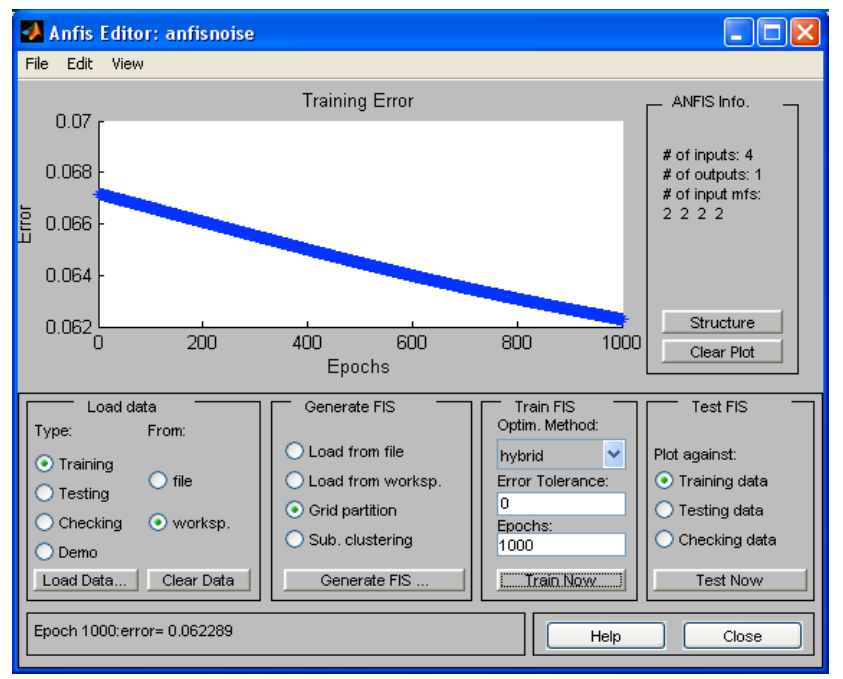

Fig. 2. Variation of training error

Neuro- fuzzy model for noise identification is composed of five componential layers. Layer №1 (input) is a layer, in which are entered the real values of the input variables.

The membership functions of the input variables are set in layer №2 (inputmf). Every membership function defines a set of values of the input variable for a specific output classification group. Transfer from real to fuzzy values is doing by 
G. Georgiev, I. Balabanova, P. Kogias, S. Sadinov and S. Kostadinova/

Journal of Engineering Science and Technology Review 11 (3) (2018) 128-132

fuzzification. For each input variable is assign calculated membership level with values ranging from 0 to 1 . The parameters setting of membership functions are performed during the training of ANFIS.

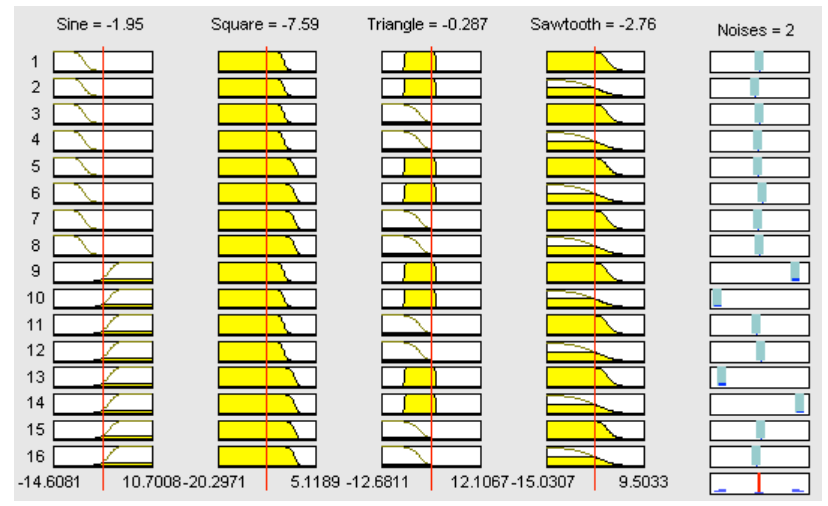

Fig. 3. Fuzzy rules

The set of fuzzy linguistic rules are applied in layer №3 (rule) and performs a process of its aggregation, in which each ordered combination of values of the input variables is assign a degree of identity to the corresponding term of the output variable. The different levels of membership are obtained for each of the terms of output variable after each linguistic rule. The output value is obtained by aggregation of these values. For noise identification the set of fuzzy rules (Fig. 3) describing all possible states of the system is equal to 16 .

After extraction of all generated rules of the neuro-fuzzy classifier in layer №4 (outputmf) are obtained the surfaces of the solutions for all possible pairs of combinations of input variables, given in Fig. 4. This is done under transition from fuzzy to the real values of the output variables.

The final result in real form is performed at the output of layer №5 (output).

\subsection{Testing of neuro-fuzzy classifier}

A procedure for ANFIS classifier testing are applied with data from the training sample and test patterns for both classes presented by Fig. 5 to Fig. 7. The obtained results for Average Testing errors are 0.062286; 0.065081 and 0.033279 , respectively for training and test data from first and second grade..

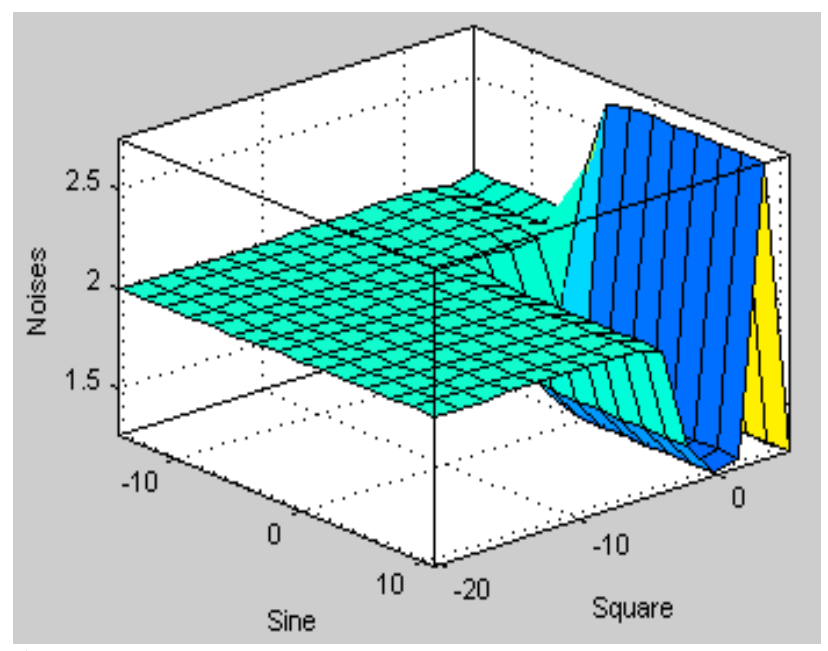

a)

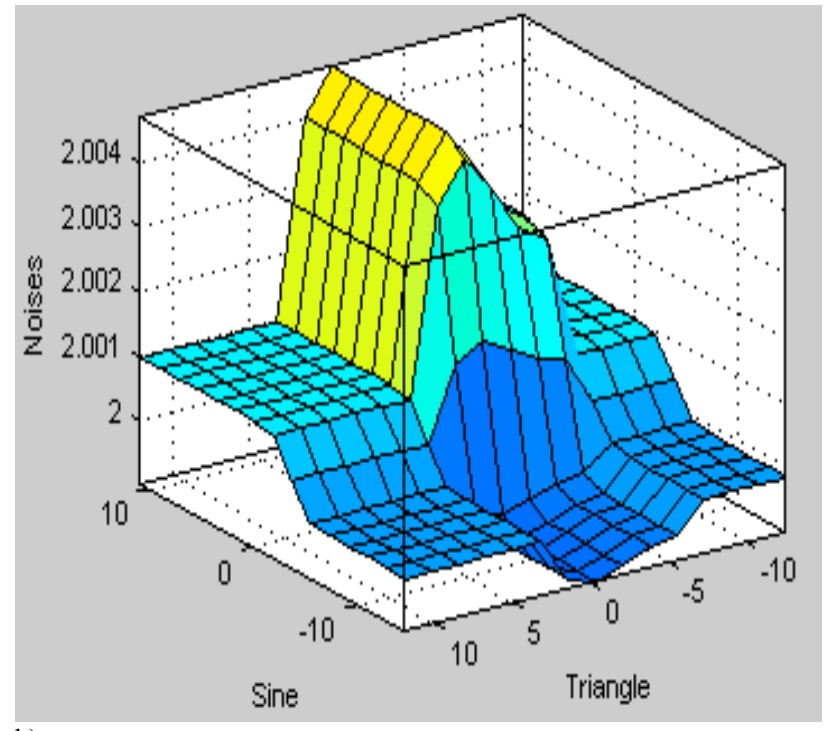

b)

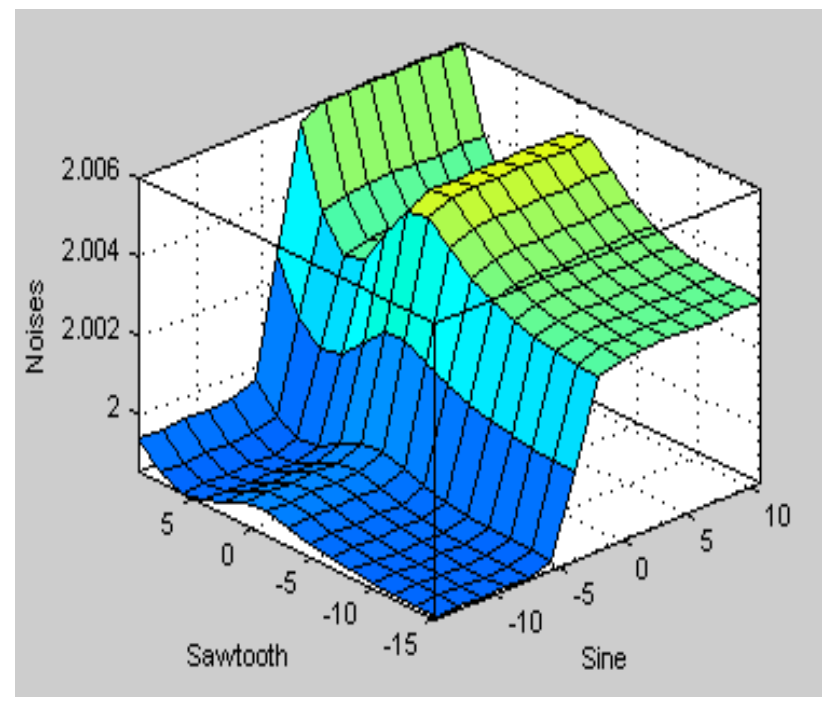

c)

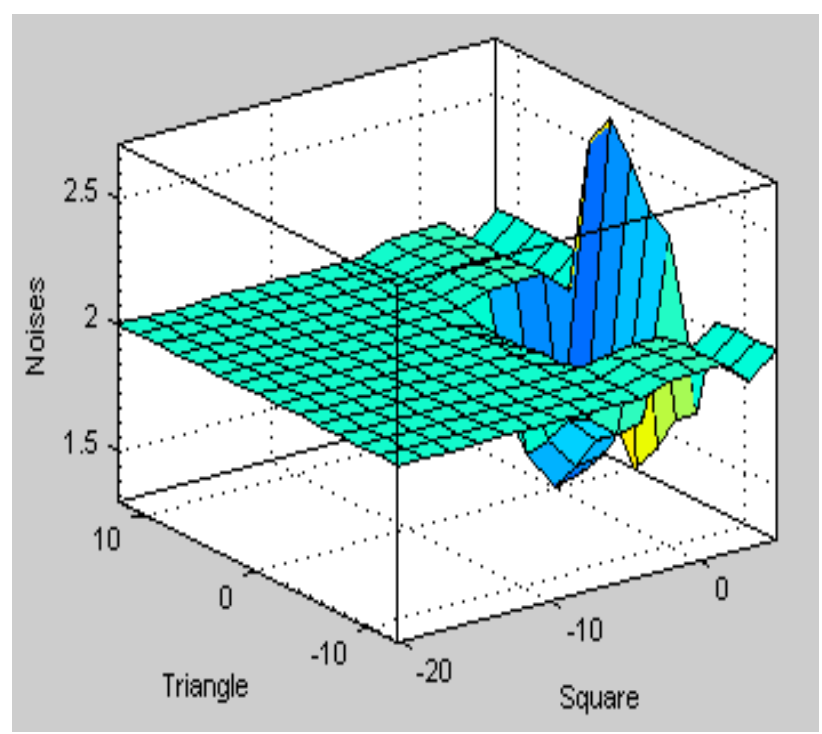

d) 
G. Georgiev, I. Balabanova, P. Kogias, S. Sadinov and S. Kostadinova/

Journal of Engineering Science and Technology Review 11 (3) (2018) 128-132
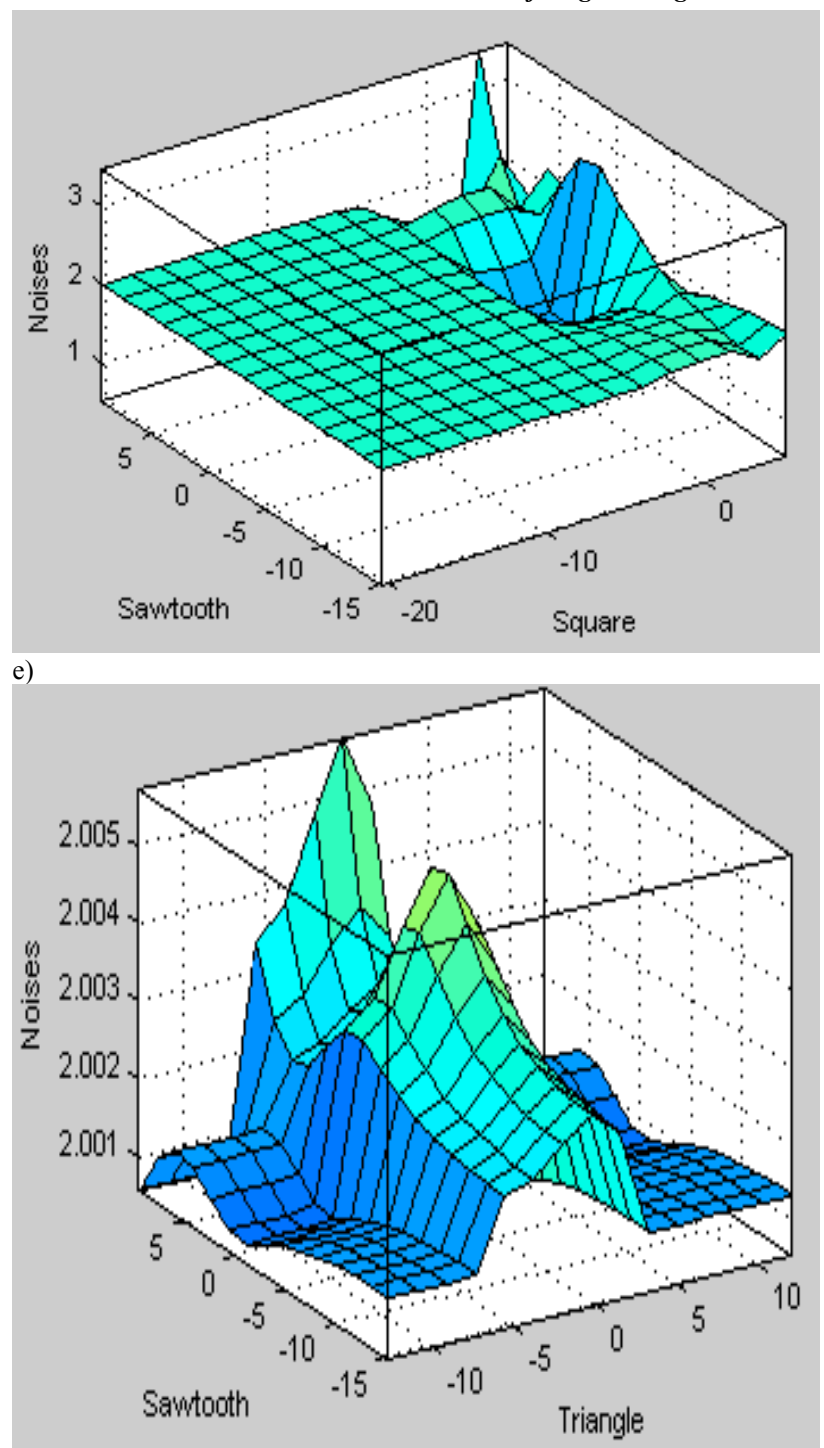

f)

Fig. 4. Decision surfaces for a) Square and Sine, b) Triangle and Sine, c) Sine and Sawtooth, d) Square and Triangle e) Square and Sawtooth and f) Triangle and Sawtooth

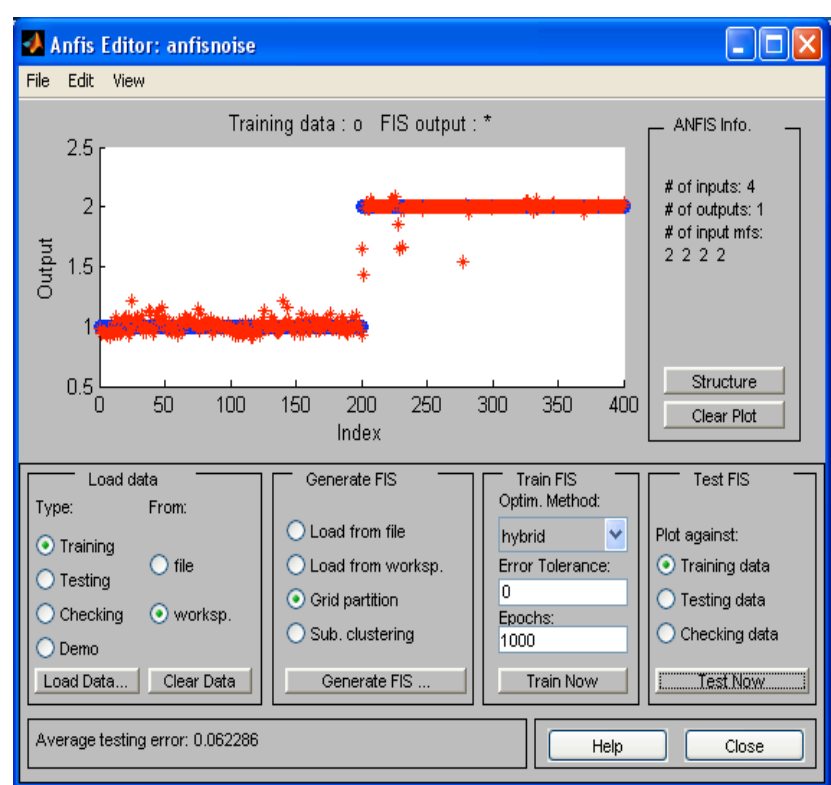

Fig. 5. The ANFIS test with test patterns from training sample

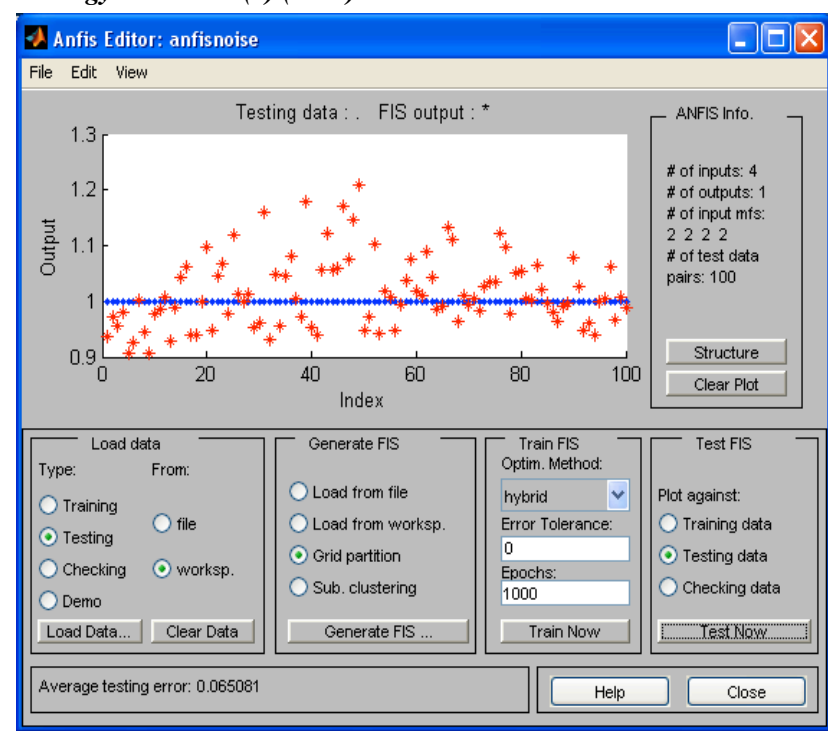

Fig. 6. The ANFIS test with test patterns from training sample for class №1

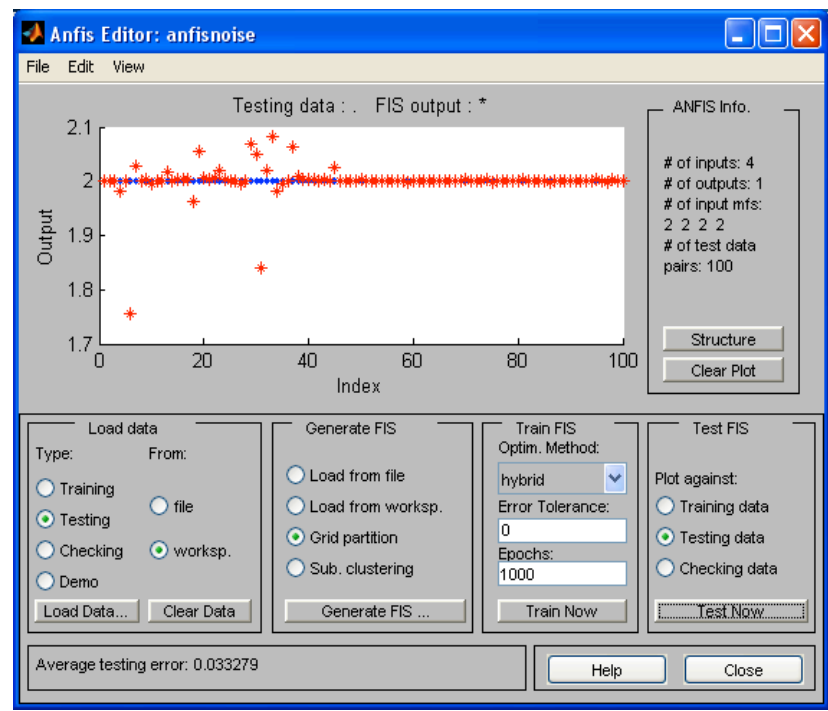

Fig. 7. The ANFIS test with test patterns from training sample for class №2

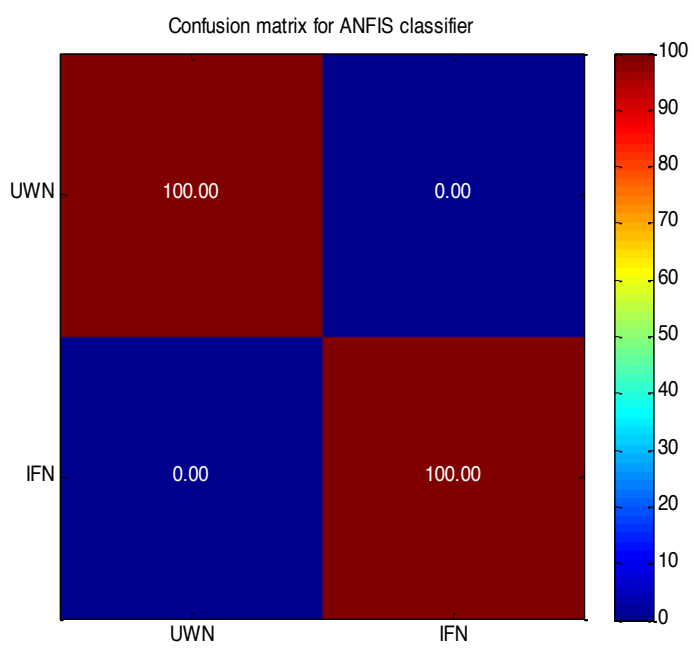

Fig. 8. Confusion matrix at testing of ANFIS 
G. Georgiev, I. Balabanova, P. Kogias, S. Sadinov and S. Kostadinova/

Journal of Engineering Science and Technology Review 11 (3) (2018) 128-132

A matrix of correct and incorrect classifications (Fig. 8) is formed for the patterns included in the composition of the test sample. The achieved classification accuracy is equial to $100.00 \%$.

3D presentation of absolute errors on figure 9 shows, that their variation does not exceed acceptable levels for a correct classification from -0.5 to +0.5 when determining the membership of the test samples.

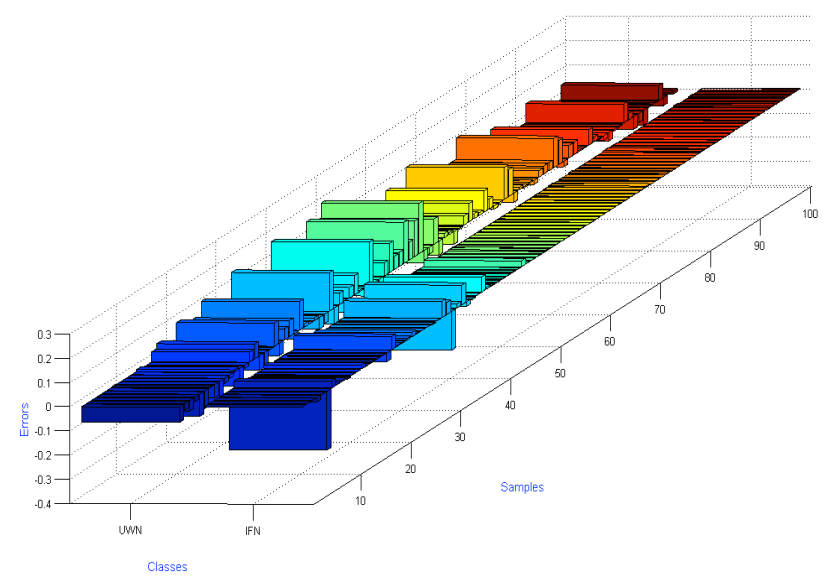

Fig. 9. Absolute errors

\section{Conclusion}

Successful identification of noise in the measurement signals in the information tele-metering systems for industrial use, reflecting the actual conditions of the managed objects and technological processes, is an important task for insuring the quality of service of maintenance systems for transmission and processing of data.

In the present study the application of Sugeno fuzzy interface systems using neural fuzzy technical approaches for solving the problem of identification of signals different in shape with superimposed Uniform white and Inverse F noises, is achieved maximum accuracy in the analysis of the test data.

The obtained results give a reason for successful implementation and application of the selected ANFIS structure as a subsystem in handling noisy signals. For the next stage of the development are planned the study the possibility of combining ANFIS architecture for noise identification with ANN and ANFIS models for adaptive filtering of signals in telecommunications.

This is an Open Access article distributed under the terms of the Creative Commons Attribution License

\section{References}

1. Chen-Chun L., Chun-Ling L., Shou J., Chin-Teng L.: The ANFIS system for nonlinear combined forecasts in the telecommunications industry., International journal of computer applications. Vol. 37, pp. 30-35 (2012)

2. Ivanova E., Iliev T., Mihaylov Gr., Stoyanov I., Tsvetanov F., Otsetova E., Radev D.: Computer modeling and simulation of teletraffic models for $5 \mathrm{G}$ networks. 38 International convention on information and telecommunication technology. Electronics and microelectronics-(MIPRO), Croatia, pp. 479-482 (2015)

3. Gite A., Bodade R., Raut B.: ANFIS controller and its application. Internatianal journal of engineering research \& technology. Vol. 2, pp. 1-5 (2013)

4. Hussian R., Massoud R., Al-Mawaldi M.: ANFIS-PID control FESsupported sit-to-stand in paraplegics: (Simulation study). Biometrical science and engineering. Vol. 7, pp. 208-217 (2014)

5. AL-Saedi M., Wu H., Handroos H.: ANFIS and fuzzy tuning of PID controller for trajectory tracking of a flexible hydraulically driven parallel robot machine. Journal of automation and control engineering. Vol. 1, pp. 70-77 (2013)

6. Funsten B.: ECG classification with an adaptive neuro-fuzzy interface system. A thesis presented to Faculty of California Polytechnic State University. pp. 1-171 (2015)

7. Nazmy T., El-Messiry H., Al-Bokhity B.: Adaptive neuro-fuzzy interface system for classification of ECG signals. Journal of theoretical and applied information technology (JATIT), pp. 71-76 (2009). http://www.jatit.org

8. Homaeinezhad H., Tavakkoli E., Atyabi S., Ghaffari A.: NeuroANFIS architecture for ECG rhythm-type recognition using different QRS geometrical-based features. Indian journal of electrical \& electronic engineering. Vol. 7, No. 2, pp. 70-83 (2011)

9. Ebrahimi A., Addeh J.: Classification of ECG arrhythmias using adaptive neuro-fuzzy interface system and cuckoo optimization algorithm. Computational research progress in applied science \& engineering (CRRASE). Vol. 01 (04), pp. 134-140 (2015)
10. Sankar B., Kumar D., Seethalakshmi K.: A new self-adaptive neuro fuzzy interface system for the removal of non-linear artifacts from the respiratory signal. Journal of computer science 8 (5). pp. 621631 (2013). Science Publications

11. Sankar A., Madhusudhanan N., Selvi J.: Removal of noise through ANFIS filtering technique (ICIRES15), pp. 1-7 (2015)

12. Anand V., Shah Sh., Kumar S.: Intelligent adaptive filtering for noise cancellation. International journal of advanced research in electrical, electronics and instrumentation engineering (IJAREEIE). Vol. 2, Issue 5, pp. 2029-2039 (2013). http://www.ijareeie.com

13. Shanmugapriya N., Chandra E.: Evaluation of sound classification using modified classifier and speech enhancement using ICA algorithm for hearing AID application. Ictact jouunal on communication technology. Vol. 6, Issue 01, pp. 1279-1288 (2016)

14. Bhattacharyya S., Basu D., Tibarewala L.: Interval type-2 fuzzy logic base multiclass ANFIS algorithm for real-time EEG based movement control of a robot arm. Robotics and autonomous systems 38, pp. 104-115 (2015). ELSEVIER

15. Ankishan H., Yilmaz D.: Comparison of SVM and ANFIS for snore related sounds classification by using the largest Lyapunov exponent and entropy. Computational and mathematical methods in medicine, pp. 1-13 (2013). Hindawi Publishing Corporation

16. Shamshirband S., Petkovic D., Hashim R., Motamedi Sh.: Adaptive neuro-fuzzy methodology for noise assessment of wind turbine. PLoS ONE 9(7): e103414. pp. 1-9 (2014). http://www.plosone.org

17. Sharma A., Vijay R., Bodhe G., Malik L.: Adaptive neuro-fuzzy interface system for traffic noise prediction. International journal of computer applications (0975-8887). Vol. 98, No. 13, pp. 14-19 (2014). http://www.ijcaonline.org

18. Koleva D., Kogias P., Comparative Analysis of Multiplied Models for Traffic Distribution in a Celular Network. Journal Of Engineering Science And Technology Review, Kavala, Greece, ISSN:17912377 ,Vol. 9 Issue 1, p52-55. 4p 